\title{
Observational Signatures of the First Galaxies
}

\author{
Jarrett L. Johnson \\ Max-Planck-Institut für extraterrestrische Physik, Giessenbachstraße, 85748 Garching, Germany \\ Theoretical Modeling of Cosmic Structures Group
}

\begin{abstract}
Detection of the radiation emitted from some of the earliest galaxies will be made possible in the next decade, with the launch of the James Webb Space Telescope (JWST). A significant fraction of these galaxies may host Population (Pop) III star clusters. The detection of the recombination radiation emitted by such clusters would provide an important new constraint on the initial mass function (IMF) of primordial stars. Here I review the expected recombination line signature of Pop III stars, and present the results of cosmological radiation hydrodynamics simulations of the initial stages of Pop III starbursts in a first galaxy at $z \sim 12$, from which the timedependent luminosities and equivalent widths of IMF-sensitive recombination lines are calculated. While it may be unfeasible to detect the emission from Pop III star clusters in the first galaxies at $z$ $>10$, even with next generation telescopes, Pop III star clusters which form at lower redshifts (i.e. at $z<6$ ) may be detectable in deep surveys by the JWST.
\end{abstract}

Keywords: cosmology, galaxy formation, H II regions, halos, high-redshift, intergalactic medium PACS: 98

\section{INTRODUCTION}

In this contribution, I will address three key questions pertaining to the observational signatures of the first galaxies, and in particular to the prospects for the detection and identification of Pop III stars. These questions are the following:

- How can Population (Pop) III stars be identified observationally and their initial mass function (IMF) constrained?

- How long did Pop III star formation continue after the epoch of the first stars?

- Will observational facilities in the coming years, and in particular the James Webb Space Telescope (JWST), be able to detect and identify Pop III stellar populations?

The next three sections are devoted to addressing these three questions in the order given above; in the final section I will briefly summarize the main conclusions.

\section{THE SPECTRAL SIGNATURE OF POP III STAR FORMATION}

It is well-known that Pop III stars are likely to be considerably hotter than present-day stars, for a given stellar mass (e.g. [3, 6, 9, 10]). The high surface temperatures of Pop III stars lead to enhanced emission of ionizing radiation, in particular photons with energies above $54.4 \mathrm{eV}$ which can ionize He II. This, in turn, implies that the photoionized regions surrounding Pop III stars should shine brightly in He II recombination lines, principally in He II $\lambda 1640$ [5, 22, 29, 25]. Indeed, a number of observational efforts have been 

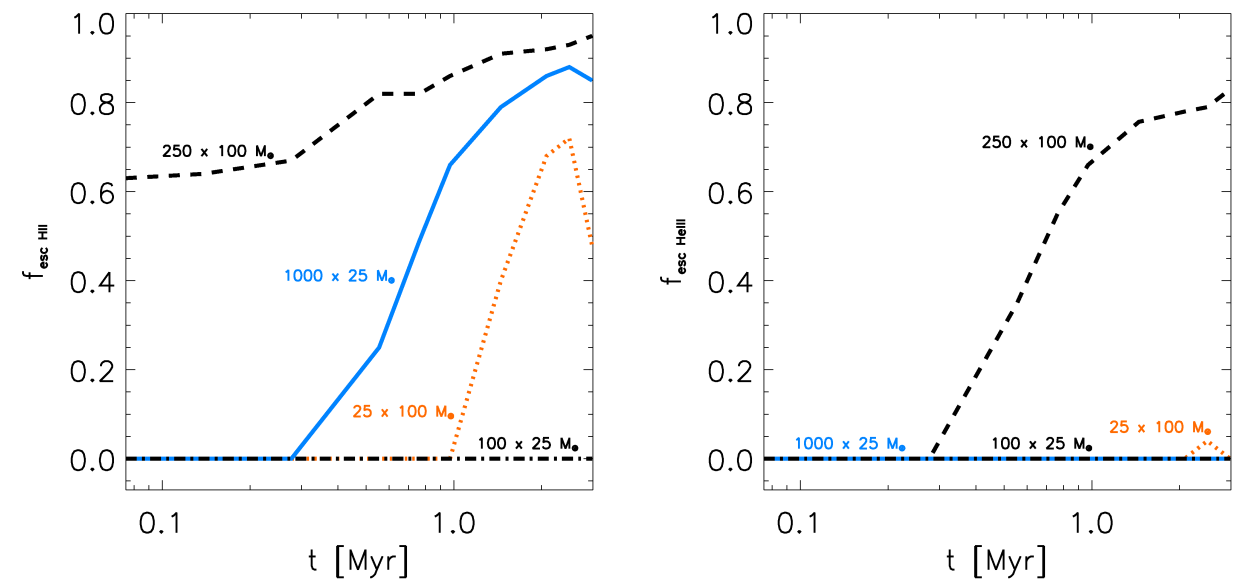

FIGURE 1. The escape fraction of $\mathrm{H}$ I-ionizing photons (left panel) and that of He II-ionizing photons (right panel), from a Pop III star cluster formed in a first galaxy at $z \sim 12$. Each line corresponds to a different choice of IMF and total number of stars, as labeled. There is a tight anticorrelation between the escape fraction of $\mathrm{H}$ I-ionizing photons and the emission in hydrogen recombination lines (e.g. Ly $\alpha$ and $\mathrm{H} \alpha$; see Figs. 2 and 3). For most cases, however, the negligible escape fraction of He II-ionizing photons leads to a tight correlation between the luminosity emitted in the He II $\lambda 1640$ line and the total mass contained in stars, for a given IMF (see [17]).

geared toward detecting this recombination line as an indicator of Pop III star formation $[19,21]$ (see also $[4,7,11,23,27,30]$ ), although to date no definitive detections on Pop III star formation have been reported. Nonetheless, the He II $\lambda 1640$ signature is likely one of the most reliable indicators of metal-free star formation and will continue to be sought using future telescopes (see e.g. [2]).

Helium recombination lines also afford a means to constrain the IMF of Pop III stars, potentially allowing to test different theoretical predictions for the characteristic mass of Pop III stars (e.g. of the order of $10 \mathrm{M}_{\odot}$ or $100 \mathrm{M}_{\odot}$ ). However, constraining the IMF using the ratio of the observed fluxes in He II $\lambda 1640$ and $\mathrm{H} \alpha$ or Ly $\alpha$ poses some challenges, owing to the evolution of the flux emitted in these lines. While the evolution of the massive stars in a cluster will alter its spectral characteristics $[25,26]$, the photoheating of the gas surrounding the cluster will also lead to similar evolution [17]. In particular, as the gas surrounding the cluster is photoheated it expands, thereby allowing for the escape of ionizing photons into the intergalactic medium (IGM); as more ionizing photons escape, fewer are available to ionize the dense gas from which recombination lines are emitted, and the luminosity in those lines correspondingly drops.

The escape fraction of ionizing photons from Pop III stellar clusters forming in a first galaxy at $z \sim 12$, as calculated from the simulations presented in [17], are shown in Figure 1. As this Figure shows, the escape fractions of H I- and He II-ionizing photons can differ greatly, leading to evolution of the ratio of the luminosities emitted in $\mathrm{H}$ I and He II recombination lines, thus complicating the use of such ratios as indicators of the IMF. For two simulations presented in [17], the evolution of the flux visible in $\mathrm{H} \alpha$ is shown in Figure 2; as the escape fraction of $\mathrm{H}$ I-ionizing photons generally increases 


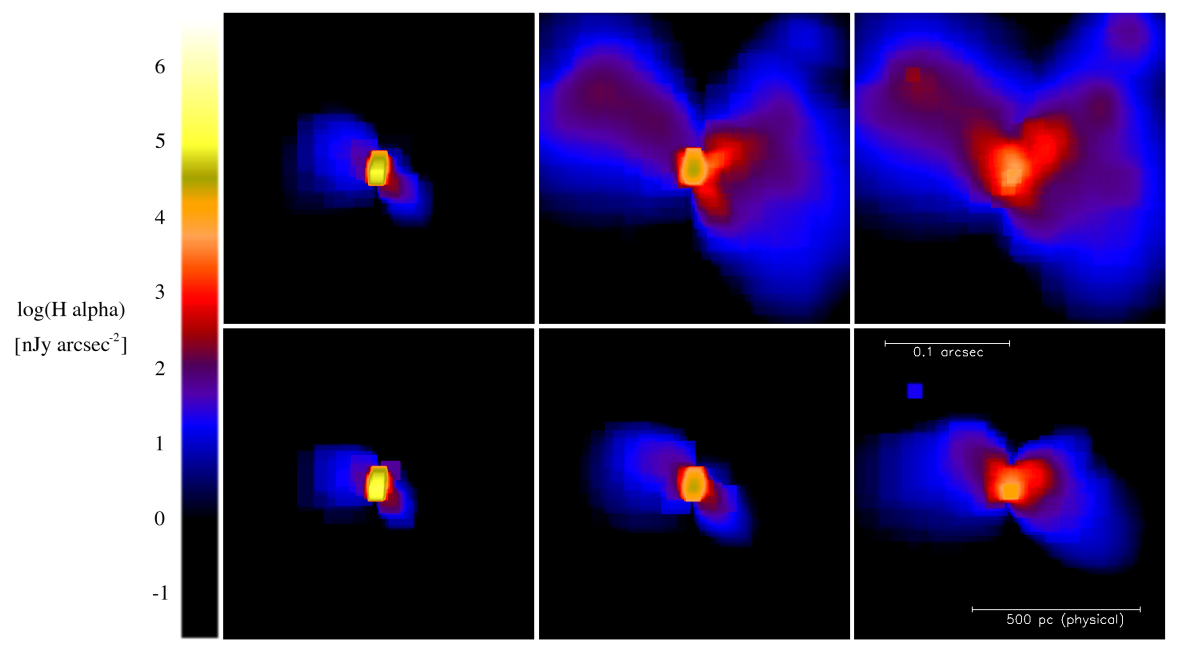

FIGURE 2. The flux in $\mathrm{H} \alpha$ per square arcsecond, emitted from Pop III stellar clusters at $z \sim 12$, as observed on the sky at $z=0$, assuming a spectroscopic resolution of $\mathrm{R}=1000$. Shown here are the two most massive of the four simulated stellar clusters presented in [17], one containing $25 \mathrm{M}_{\odot}$ stars (bottom panels), the other containing $100 \mathrm{M}_{\odot}$ stars (top panels). From left to right, the clusters are shown at $10^{5}$ $\mathrm{yr}, 1 \mathrm{Myr}$, and $3 \mathrm{Myr}$ after formation. The highest total fluxes occur at the earliest times, before the $\mathrm{H}$ II region has broken out of the galaxy; hence, the youngest stellar clusters are the most readily observed.

with time, the total flux is highest at early times, making the youngest clusters the most easily observed.

Figure 3 shows the equivalent width (EW) of three prominent recombination lines for each of the four simulations presented in [17]. While the ratio of the fluxes in He II and $\mathrm{H}$ I recombination lines can be a problematic indicator of the stellar IMF, this Figure shows that the EW of He II $\lambda 1640$ may be a more robust indicator, always being larger for clusters with the more top-heavy IMF regardless of the total mass in stars.

\section{POP III STAR CLUSTERS FORMED AFTER REIONIZATION}

It is likely that even surveys to be carried out by the JWST will not be deep enough to detect the first stars or galaxies at $z \geq 10[1,13,17,24]$, although Pop III supernovae (e.g. $[14,28,31,33])$ and perhaps stars powered by dark matter (DM) annihilation [12, 34] may still be detected. This provides motivation to consider whether Pop III star clusters may form also at lower redshifts, where IMF-sensitive recombination lines may be more readily detected.

In regions of the universe that undergo reionization at sufficiently early times (i.e. $z \sim 20$ ), thereby quenching star formation in DM minihaloes, Pop III star clusters may form in the unenriched descendants of those minihalos at $z \leq 6$. Figure 4 shows the predicted abundance of such clusters, for various assumptions on the reionization history, the minimum mass of halos which may host star formation after reionization, and the speed of external metal enrichment by neighboring galaxies [16]. As this Figure shows, although likely to be very rare, such Pop III clusters may be abundant enough to 


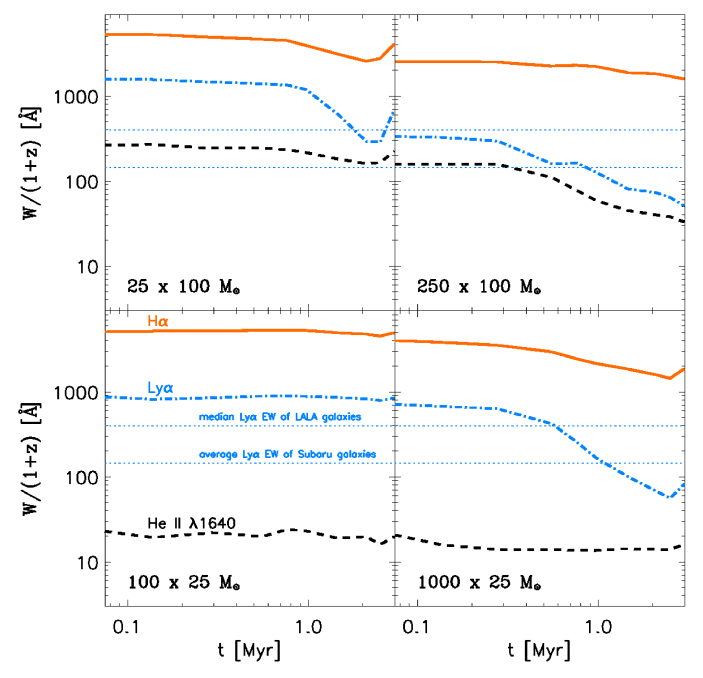

FIGURE 3. The rest frame EWs of $\operatorname{Ly} \alpha, \mathrm{H} \alpha$, and He II $\lambda 1640$, as a function of time, for the same clusters as shown in Figs. 1 and 2. The dotted lines show the observed EWs of galaxies from two different surveys carried out at $z=4.5$ and $z \geq 6$ ([18], [20]). The He II $\lambda 1640 \mathrm{EW}$ for clusters of $100 \mathrm{M}_{\odot}$ stars is always higher than that for $25 \mathrm{M}_{\odot}$ stars, regardless of the total stellar mass in the clusters; hence, we conclude that the EW of this line is a robust indicator of a very top-heavy IMF (see [17]).

be detected in the Deep-Wide Survey (DWS) to be carried out by the JWST [13, 32].

\section{PROSPECTS FOR DETECTION IN JWST DEEP SURVEYS}

If the number density of Pop III stellar clusters at low redshift (e.g. $z \leq 6$ ) is indeed high enough for some of them to lie within the area to be surveyed by the JWST, the question remains whether these clusters would be bright enough to be detected. Figure 5 shows the monochromatic $\operatorname{Ly} \alpha$ flux predicted for such clusters, for different assumptions on the IMF, the minimum halo mass for star formation, and the star formation efficiency [16]. As the Figure shows, for a very top-heavy IMF and/or a high star formation efficiency, the JWST NIRCam may detect these clusters in the planned DWS. Furthermore, spectroscopic follow-up with NIRSpec may detect the He II $\lambda 1640$ flux, thereby allowing both for the confirmation of candidate Pop III star clusters and for constraints to be placed on the stellar IMF [16] (see also [8]).

It should be noted that strong He II $\lambda 1640$ emission, while a telltale sign of Pop III star formation, does not in itself prove the existence of Pop III stars. Other observational signatures of Pop III star formation should thus be pursued. Among the other signs of Pop III star formation may be a distinct absence of metal emission or absorption lines. Additionally, unless the mixing of metals with the primordial gas is sufficiently slow (see e.g. [15]), one would expect Pop III star clusters not to form in galaxies in which previous star formation and metal enrichment have occurred; instead, as discussed above, Pop III star formation may occur only in previously unenriched DM halos at $z \leq$ 6 , and furthermore perhaps only in halos within typical distances of $\sim 1 \mathrm{Mpc}$ comoving of galaxies which begin reionizing the IGM at $z \sim 20[16]$. 


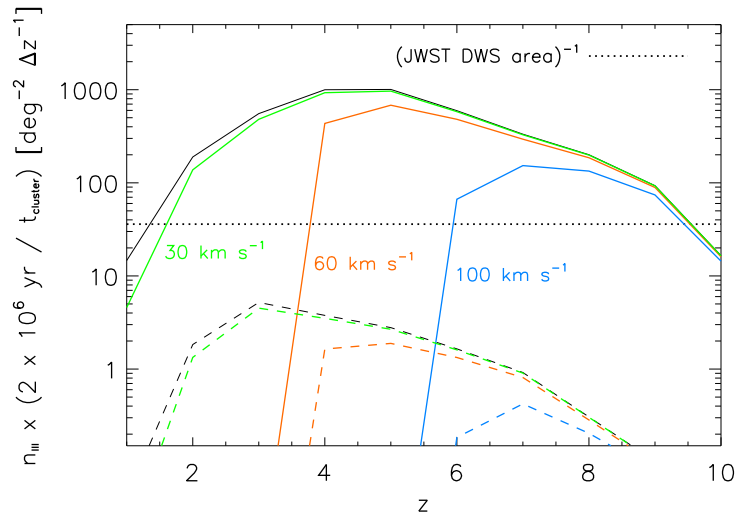

FIGURE 4. The number density $n_{\text {III }}$ of Pop III star clusters formed in reionized regions of the universe, taking into account enrichment of the IGM by galactic winds, and normalized to a cluster lifetime $t_{\text {cluster }}$ $=2 \mathrm{Myr}$. The solid lines correspond to a minimum halo circular velocity for star formation of $20 \mathrm{~km} \mathrm{~s}^{-1}$, while the dashed lines correspond to a minimum of $30 \mathrm{~km} \mathrm{~s}^{-1}$. For each series of lines, the top (black) line is a model which neglects external metal enrichment, while the colored lines correspond to different metal-enriched wind velocities, as labeled. The number density at which one cluster per unit redshift is expected to be within the planned JWST Deep-Wide Survey area is shown by the dotted line (see [16]).

\section{SUMMARY}

In closing, I would like to highlight the following conclusions corresponding to the three key questions addressed in the work presented here:

- The Pop III IMF can be constrained with detection of helium recombination emission (particularly the He II $\lambda 1640$ line). This emission varies due to both stellar evolution and hydrodynamic evolution of photoionized regions.

- In rare regions which are reionized at early times, Pop III star formation may extend well beyond the epoch of the first stars and galaxies.

- If this is so, then planned JWST surveys may detect Pop III stellar clusters at redshifts $z<6$ and allow for constraints to be placed on the IMF.

\section{ACKNOWLEDGMENTS}

I would like to thank the conference organizers, Dan Whalen, Naoki Yoshida, and Volker Bromm, for hosting a most enjoyable and productive event, as well as for allowing me to present this work. I am also grateful for support from the Theoretical Modeling of Cosmic Structures (TMoX) Group at MPE.

\section{REFERENCES}

1. Barkana R., Loeb A. 2000, ApJ, 531, 613

2. Barton E. J., Davé R., Smith J.-D. T., Papovich C., Hernquist L., Springel V. 2004, ApJ, 604, L1 


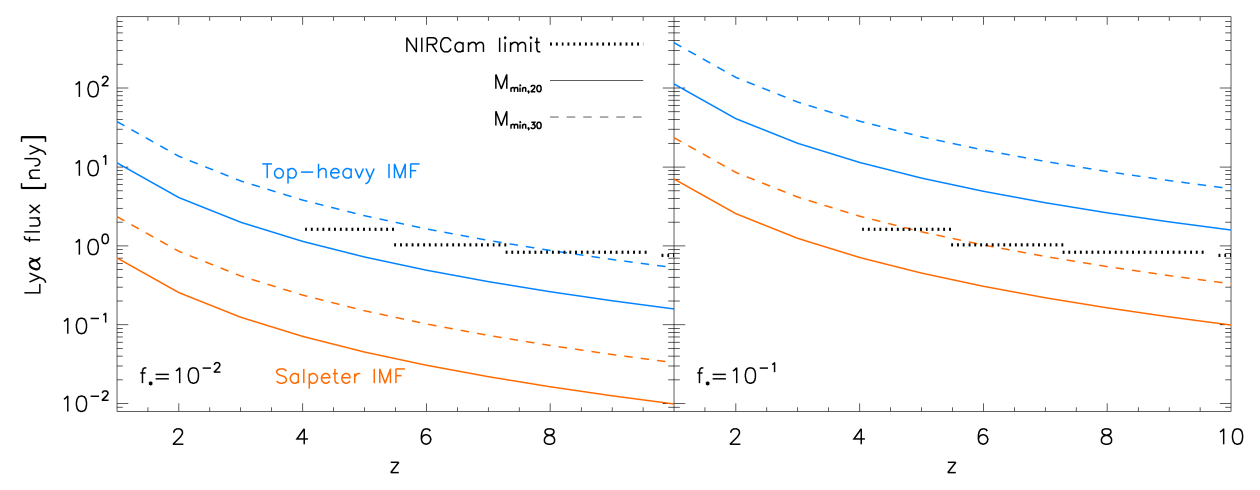

FIGURE 5. The monochromatic Ly $\alpha$ flux of Pop III clusters as would be observed by the NIRCam instrument in the JWST DWS, for different choices of the IMF, star formation efficiency $f_{*}$, and minimum halo mass $M_{\min }$ for star formation, as described in [16]. The black dotted lines are the $3 \sigma$ detection limits expected for the DWS, for an exposure time of $2 \times 10^{5}$ seconds. For a star formation efficiency of $f_{*}$ $=10^{-2}$ (left panel) only clusters with a top-heavy IMF may be detected, while for a high star formation efficiency $f_{*}=10^{-1}$ (right panel) even clusters with a Salpeter IMF may be detectable out to $z \sim 6$.

3. Bond J. R., Arnett W. D., Carr B. J. 1984, ApJ, 280, 825

4. Bouwens R. J., et al. 2009, ApJ, submitted (arXiv:0910.0001)

5. Bromm V., Kudritzki R. P., Loeb A. 2001, ApJ, 552, 464

6. Castellani V., Chieffi A., Tornambe A. 1983, ApJ, 272, 249

7. Dawson S., et al. 2004, ApJ, 617, 707

8. Dijkstra M., Lidz A., Wyithe J. S. B. 2007, MNRAS, 377, 1175

9. $\quad$ El Eid M. F., Fricke K. J., Ober W. W. 1983, A\&A, 119, 54

10. Ezer D. \& Cameron A. G. W. 1971, Ap\&SS, 14, 399

11. Fosbury R. A. E., et al. 2003, ApJ, 596, 797

12. Freese K., Ilie C., Spolyar D., Valluri M., Bodenheimer P. 2010, arXiv:1002.2233

13. Gardner J. P., et al. 2006, SSRv, 123, 485

14. Haiman Z. 2008, Astrophysics in the Next Decade: JWST and Concurrent Facilities, Ap\&SS Library, Eds. H. Thronson, A. Tielens, M. Stiavelli (arXiv:0809.3926)

15. Jimenez R., Haiman Z. 2006, Nat, 440, 501

16. Johnson J. L. 2010, MNRAS, in press (arXiv:0911.1294)

17. Johnson J. L., Greif T. H., Bromm V., Klessen R. S., Ippolito J. 2009, MNRAS, 399, 37

18. Malhotra S., Rhoads J. E. 2002, ApJ, 565, L71

19. Nagao T., et al. 2005, ApJ, 631, L5

20. Nagao T., et al. 2007, A\&A, 468, 877

21. Nagao T., et al. 2008, ApJ, 680, 100

22. Oh P., Haiman Z., Rees M. J. 2001, ApJ, 553, 73

23. Prescott M. K. M., Dey A., Jannuzi B. T. 2009, ApJ, 702, 554

24. Ricotti M., Gnedin N. Y., Shull J. M. 2008, ApJ, 685, 21

25. Schaerer D. 2002, A\&A, 382, 28

26. Schaerer D. 2003, A\&A, 397, 527

27. Shapley A. E., Steidel C. C., Pettini M., Adelberger K. L. 2003, ApJ, 588, 65

28. Trenti M., Stiavelli M., Shull J. M. 2009, ApJ, 700, 1672

29. Tumlinson J., Giroux M. L., Shull J. M. 2001, ApJ, 550, L1

30. Wang J. X., Malhotra S., Rhoads J. E., Zhang H. T., Finkelstein S. L. 2009, ApJ, 706, 762

31. Weinmann S. M. \& Lilly S. J. 2005, ApJ, 624, 526

32. Windhorst R. A., Cohen S. H., Jansen R. A., Conselice C., Yan H. 2006, New Astron. Rev., 50, 113

33. Wise J. H. \& Abel T. 2005, ApJ, 629, 615

34. Zackrisson E., et al. 2010, ApJ, submitted (arXiv:1002.3368) 